\title{
Runaway Greenhouse Effect in a Semigray Radiative-Convective Model
}

\author{
T. Pujol and G. R. NORTH \\ Department of Atmospheric Sciences, Texas A\&M University, College Station, Texas
}

(Manuscript received 22 January 2001, in final form 27 February 2002)

\begin{abstract}
The effects of the nongray absorption (i.e., atmospheric opacity varying with wavelength) on the possible upper bound of the outgoing longwave radiation (OLR) emitted by a planetary atmosphere have been examined. This analysis is based on the semigray approach, which appears to be a reasonable compromise between the complexity of nongray models and the simplicity of the gray assumption (i.e., atmospheric absorption independent of wavelength). Atmospheric gases in semigray atmospheres make use of constant absorption coefficients in finite-width spectral bands. Here, such a semigray absorption is introduced in a one-dimensional (1D) radiativeconvective model with a stratosphere in radiative equilibrium and a troposphere fully saturated with water vapor, which is the semigray gas. A single atmospheric window in the infrared spectrum has been assumed.

In contrast to the single absolute limit of OLR found in gray atmospheres, semigray ones may also show a relative limit. This means that both finite and infinite runaway effects may arise in some semigray cases. Of particular importance is the finding of an entirely new branch of stable steady states that does not appear in gray atmospheres. This new multiple equilibrium is a consequence of the nongray absorption only. It is suspected that this new set of stable solutions has not been previously revealed in analyses of radiative-convective models since it does not appear for an atmosphere with nongray parameters similar to those for the earth's current state.
\end{abstract}

\section{Introduction}

The heat engine corresponding to the general circulation of the climate in a terrestrial planet is essentially determined from the balance between the outgoing longwave radiation (OLR) and the absorbed sunlight. In general, atmospheric gases may attenuate both long- and shortwave energy fluxes and emit longwave radiation only. Then, it is reasonable to expect a vertical variation of radiative fluxes throughout the atmosphere. In addition, convective processes may originate at the surface of the planet, so that material (i.e., nonradiative) energy fluxes may also propagate vertically. Because of these features, one-dimensional vertical models have played an important role in the analysis of the global climate by means of simple mathematical models.

Pioneering studies of one-dimensional vertical atmospheres dealt with the gray absorption assumption (i.e., equal absorption at all wavelengths), being applied to planetary atmospheres (e.g., Simpson 1927), as well as to stellar interiors (e.g., see Chandrasekhar 1960, and the references therein). Pure radiative equilibrium atmospheres especially designed for the earth's current state were later developed (e.g., Manabe and Möller 1961), where the absorption of several atmospheric gases was expressed in terms of mean absorptivity param-

Corresponding author address: Dr. T. Pujol, Dept. de Física, Universitat de Girona, Campus Montilivi, Girona 17071, Catalonia, Spain. E-mail: toni.pujol@udg.es eters and transmissivity functions. The introduction of convective processes was first based on an empirical technique, which assumed a constant lapse rate within the convective layer (Manabe and Strickler 1964; Manabe and Wetherald 1967). Semiempirical techniques for expressing the physics of convection in one-dimensional radiative-convective models have also been developed (e.g., Ramanathan and Coakley 1978; Lindzen et al. 1982; Emanuel 1994, and the references therein).

For quantitative analyses, the gray assumption was clearly rejected in favor of detailed nongray calculations requiring numerical solutions (e.g., Arking 1972; Houghton 1986). Recently, however, the gray assumption has been adopted in the development of comprehensive radiative-convective models focused on understanding the role of convective processes in the global climate (e.g., $\mathrm{Hu}$ and Randall 1995; Kelly et al. 1999; Lindzen et al. 2001). Radiative-convective gray models have also been used to analyze the fundamentals of the atmospheric radiation limits (Nakajima et al. 1992) and their implications to climate dynamics (Pierrehumbert 1995). Important contributions to the subject of atmospheric radiation limits are due to Simpson (1927), Komabayasi (1967), and Ingersoll (1969), who independently found that the OLR emitted by a planetary gray atmosphere fully transparent to sunlight could not exceed a given upper bound (hereafter called SKI limit).

The existence of the SKI limit is of particular importance when analyzing planetary evolution problems 
(e.g., Pollack 1971; Kasting et al. 1984; Kasting 1988) since, in equilibrium, the OLR is equivalent to the absorbed solar radiation and the solar flux increases with time from the theory of star evolution (see, e.g., Crowley and North 1991). For a value of the absorbed solar radiation below the SKI limit, the condensable and absorbing gas in a gray planetary atmosphere is in equilibrium with its liquid (or solid) phase at the surface (see Ingersoll 1969). In contrast, for a value of the absorbed solar radiation exceeding the SKI limit, the amount of vapor is so high (i.e., the greenhouse effect is so intense) that the radiation emitted by the atmosphere cannot balance that absorbed, and the vaporliquid (or vapor-solid) equilibrium at the surface is not possible. Then, the liquid phase would evaporate entirely. Hence, the SKI limit is known as the runaway greenhouse point.

The existence of an atmospheric radiation limit (i.e., of an upper bound in OLR) may also imply a multiplicity of steady states. Note that we may plot the OLR as a function of the surface temperature. Since, in most cases, the SKI limit is reached at a finite temperature, we may expect two different values of surface temperature, at least, for the same value of OLR ( $<$ SKI limit). Multiple equilibria are a common feature of simple vertically averaged latitude-dependent climate models with ice albedo feedback (e.g., North et al. 1981). In onedimensional radiative-convective models, however, the multiplicity of steady states is rare to find. $\mathrm{Li}$ et al. (1997) have recently shown that multiple equilibria arise in a simple one-dimensional pure radiative vertical model with ice albedo feedback. Of more fundamental interest are the multiple equilibria found by Rennó (1994, 1997) in radiative-convective models with an explicit hydrological cycle, and the bimodal solutions in a pure radiative model found by Ide et al. (2001).

The purpose of the present paper is to revisit the thorough analysis of the runaway greenhouse effect (i.e., on the fundamentals of the SKI limit) carried out by Nakajima et al. (1992) by introducing a basic nongray (semigray) absorption in the way suggested by Simpson (1928) and Weaver and Ramanathan (1995) and defined in section 2. We make use of a simple nongray formulation, since complex nongray models are difficult to interpret and may hide the intrinsic behavior of the system. Note that the semigray approach employed here would be only of merely academic interest if the results were not substantially different from the gray model assumption. This is not the case since radiation limits due to either stratospheric (section 3) or tropospheric (section 4) conditions indicate the possibility of a finite runaway effect not found in gray atmospheres that may have implications in the theory of planetary evolution. In addition, we have found an entirely new set of multiple equilibria not found in previous one-dimensional vertical models that arises from the inclusion of the nongray absorption only (see the discussion in section 5). The results are summarized in section 6 .

\section{Simple nongray model}

For simplicity, we assume a plane-parallel atmosphere completely transparent to shortwave radiation and divided into two layers. The upper layer, or stratosphere, is assumed to be in radiative equilibrium whereas the lower layer, or troposphere, is in radiative-convective equilibrium. The transition level between the two layers is referred to as the tropopause. The atmosphere contains two gases only. One of them is noncondensable and fully transparent to both short- and longwave radiation, whereas the other is condensable and absorbs in the infrared with a region of low absorption (atmospheric window). We use a very crude approximation for the nongray absorption by assuming constant values of the monochromatic mass absorption coefficient $k_{v}$ of the absorbing gas in finite-width spectral bands

$$
\begin{aligned}
k_{v}= & k_{1}\left[\theta\left(v-v_{u}\right)+\theta\left(v_{l}-v\right)\right] \\
& +k_{0}\left[\theta\left(v_{u}-v\right)-\theta\left(v_{l}-v\right)\right],
\end{aligned}
$$

where $v_{l}$ and $v_{u}$ define the lower- and the upper-frequency values of the atmospheric window, and $\theta$ being Heaviside's theta function. In Eq. (1), $k_{0}$ and $k_{1}$ are constants and define the mass absorption coefficients inside and outside the atmospheric window, respectively. The gray atmosphere is obtained either with $k_{0}=k_{1}$ or $v_{l}=v_{u}$. Note that $k_{0}=0$ implies a null absorption within the atmospheric window. In this case, a fraction of the radiation emitted at the surface directly escapes to the space (see, Simpson 1928; Weaver and Ramanathan 1995).

The atmospheric opacity not only depends on the mass absorption coefficient $k_{v}(1)$, but also on the amount of the absorbing gas. Following Nakajima et al. (1992), the variation of the monochromatic optical depth $\tau_{v}$, when the specific intensity of infrared radiation $I_{v}$ of frequency $v$ crosses an atmospheric layer of thickness $d p, p$ being the pressure, is

$$
d \tau_{v}=\frac{k_{v} x_{c} m_{c}}{\bar{m} g} d p,
$$

where $x_{c}$ is the mole fraction of the absorbing (and condensable) gas $\left(=p_{c} / p, p_{c}\right.$ being the partial pressure of the condensable component), $g$ is the acceleration of gravity, and $m_{c}$ and $\bar{m}$ are the molecular weights of the condensable and averaged components, respectively.

The radiative transfer equation which determines the variation of $I_{v}$ that crosses the atmospheric layer of thickness $d p$ can be written as (see, e.g., Goody and Yung 1989)

$$
\mu \frac{d I_{v}}{d \tau_{v}}=I_{v}-B_{v},
$$

where $\mu$ is the cosine of the zenith angle, and $B_{v}$ is Planck's function. In (3), we implicitly assume local 
thermodynamic equilibrium (LTE) conditions (Kondratyev 1969).

In general, the specific intensity of radiation $I_{v}$ is a function of $\mu$. For simplicity, we apply the two-stream approximation, which assumes two hemispherically averaged angular intensities $I_{v}^{+}$(upper hemisphere) and $I_{v}^{-}$(lower hemisphere; Liou 1992). Then, the equation of radiative transfer (3) may be expressed in terms of the longwave energy flux of radiation $F_{v}\left[=2 \pi \mu_{0}\left(I_{v}^{+}-\right.\right.$ $I_{v}^{-}$), where $\mu_{0}=1 / \sqrt{3}$; see Liou 1992] and the spherically averaged intensity of radiation $J_{v}\left[=\left(I_{v}^{+}+I_{v}^{-}\right) / 2\right.$; see Liou 1992]. Equation (3), applied to both upward $I_{v}^{+}$and downward $I_{v}^{-}$streams, is then equivalent to

$$
\begin{aligned}
\frac{d F_{v}}{d p} & =\frac{4 \pi k_{v} x_{c} m_{c}}{\bar{m} g}\left(J_{v}-B_{v}\right), \\
\frac{d J_{v}}{d p} & =\frac{k_{v} x_{c} m_{c}}{4 \pi \mu_{0}^{2} \bar{m} g} F_{v},
\end{aligned}
$$

where we have employed (2) so that pressure can be used as the vertical coordinate.

Here, $F_{v}$ is constant in a stratosphere under monochromatic radiative equilibrium conditions, from which the lhs of (4) is zero (i.e., $d F_{v} / d p=0 \forall v$ ). Then, the integration of (5) over pressure leads to

$$
J_{v}=\frac{F_{v}}{4 \pi \mu_{0}^{2}}\left(\frac{k_{v} x_{c} m_{c}}{\bar{m} g} p+\mu_{0}\right),
$$

since the downward infrared intensity is zero at the top of the atmosphere [TOA; i.e., $I_{v}^{-}(p=0)=0$, or equivalently, $J_{v}(0)=F_{v} /\left(4 \pi \mu_{0}\right)$ from the text above (4)]. Note that in (6) we have assumed a constant value of $x_{c}$ in the stratosphere (Nakajima et al. 1992).

From (1), the integration of (6) over frequencies applied to the tropopause level (i.e., $p=p_{\text {tp }}$ ) yields

$J=\frac{k_{1} x_{c} m_{c} p_{\mathrm{tp}}}{4 \pi \mu_{0}^{2} \bar{m} g}\left(F-F_{w}\right)+\frac{k_{0} x_{c} m_{c} p_{\mathrm{tp}}}{4 \pi \mu_{0}^{2} \bar{m} g} F_{w}+\frac{F}{4 \pi \mu_{0}}$,

whereas that over the atmospheric window is

$$
J_{w}=\frac{F_{w}}{4 \pi \mu_{0}^{2}}\left(\frac{k_{0} x_{c} m_{c} p_{\mathrm{tp}}}{\bar{m} g}+\mu_{0}\right),
$$

where $J$ and $F$ refer to the integrated values of $J_{v}$ and $F_{v}$ over the whole infrared spectrum, whereas $J_{w}$ and $F_{w}$ refer to the integrated values of $J_{v}$ and $F_{v}$ over the atmospheric window (i.e., from $v=v_{l}$ until $v=v_{u}$ ).

On the other hand, the integration of (4) over the whole infrared spectrum yields

$$
\begin{aligned}
0= & \frac{4 \pi k_{1} x_{c} m_{c}}{\bar{m} g}\left(J-B-J_{w}+\beta_{w} B\right) \\
& +\frac{4 \pi k_{0} x_{c} m_{c}}{\bar{m} g}\left(J_{w}-\beta_{w} B\right),
\end{aligned}
$$

whereas that over the atmospheric window (i.e., from $v=v_{l}$ to $v=v_{u}$ ) gives

$$
0=\frac{4 \pi k_{0} x_{c} m_{c}}{\bar{m} g}\left(J_{w}-\beta_{w} B\right) .
$$

The coefficient $\beta_{w}$ in (9)-(10) is a measure of the effective width of the atmospheric window, defined as (see, Weaver and Ramanathan 1995)

$$
\beta_{w}=\frac{\int_{v_{l}}^{v_{u}} d v B_{v}}{\int_{0}^{\infty} d v B_{v}} .
$$

Note that the denominator of the rhs in (11) is $B=$ $\sigma T^{4} / \pi, \sigma$ being the Stefan-Boltzmann constant. Once we fix the value of the OLR $(=F)$, Eqs. (7)-(10) form a set of four equations with four unknowns $\left(J, J_{w}, F_{w}\right.$, and $B$ ). Our purpose is to solve this algebraic system for the variable $B$ (which is directly proportional to $T^{4}$ ). This is carried out in the next two sections for both dirty and clean window cases.

\section{a. Nonzero absorption within the atmospheric window}

Previous analyses of semigray atmospheres (Simpson 1928; Weaver and Ramanathan 1995; Thomas and Stamnes 1999) assume a completely transparent window (i.e., $k_{0}=0$ ). Nevertheless, the case with $k_{0} \neq 0$ is expected to represent actual conditions better since a water vapor absorption continuum is found within the atmospheric window (see, e.g., Houghton 1986). We will analyze solutions for different ratios $k_{0} / k_{1}$, since Kasting et al. (1984) point out an extreme sensitivity of the climate with respect to changes in the opacity of the atmospheric window.

The vertical temperature distribution in this case is obtained as follows. We express $F_{w}$ in terms of $J_{w}$ from (8). The expression is substituted in (7) from which we obtain a single equation in terms of $J, J_{w}$, and $F$. Note that Eq. (10) implies $J_{w}=\beta_{w} B$, from which Eq. (9) gives $J=B$. Then, Eq. (7) may express $B$ in terms of $F$ as

$$
\pi B=\frac{\left(\tau_{k} k_{1}+\mu_{0}\right)\left(\tau_{k} k_{0}+\mu_{0}\right)}{\left[\tau_{k} k_{1} \beta_{w}+\tau_{k} k_{0}\left(1-\beta_{w}\right)+\mu_{0}\right]} \frac{F}{4 \mu_{0}^{2}},
$$

where $\tau_{k}=x_{c} m_{c} p_{\text {tp }} /(\bar{m} g)$.

The classical gray result (see, e.g., Liou 1992) is obtained from (12) if $k_{0}=k_{1}$. Gray solutions are also obtained either with $\beta_{w}=0$ (mass absorption coefficient equal to $k_{1}$ ) or $\beta_{w}=1$ (mass absorption coefficient equal to $k_{0}$ ).

\section{b. Null absorption within the atmospheric window}

In this case $\left(k_{0}=0\right)$, Eq. (10) is identically satisfied. An additional equation is obtained by noting that now the flux of radiation integrated over the atmospheric 


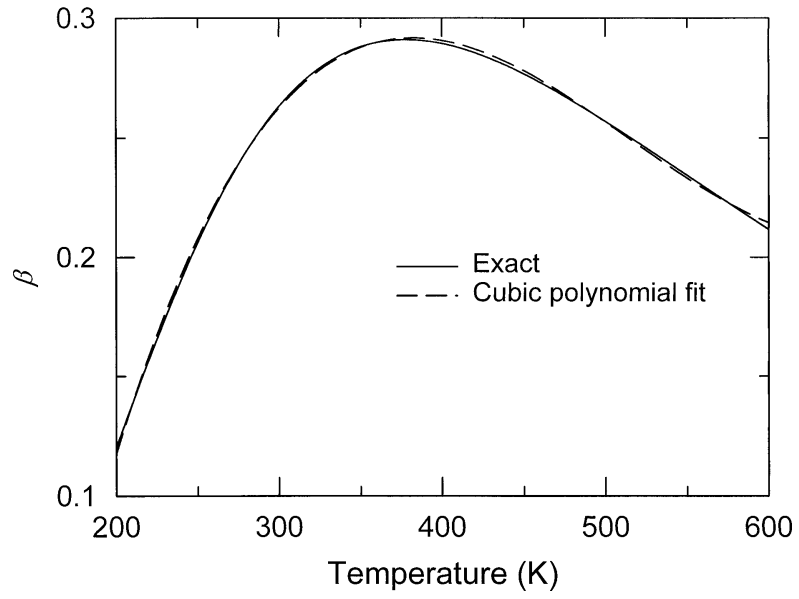

FIG. 1. Cubic polynomial fit (dashed line) to the $\beta$ function [solid line; see Eq. (11)]. Values of the coefficients are listed in the text.

window $F_{w}$ is equal to that emitted at the surface $\left[F_{w}\right.$ $=2 \mu_{0} \beta_{s w} \pi B_{s}$, where $B_{s}=B\left(T_{s}\right)$ and $\beta_{s w}=\beta_{w}\left(T_{s}\right), T_{s}$ being the surface temperature]. From this condition, and (7)-(9), we obtain

$$
\pi B=\frac{\left(\tau_{k} k_{1}+\mu_{0}\right)}{\left(1-\beta_{w}\right)}\left(\frac{F}{4 \mu_{0}^{2}}-\frac{\beta_{s w} \pi B_{s}}{2 \mu_{0}}\right) .
$$

The tropopause temperature in an atmosphere with a clear window is not only a function of the OLR $F$ (or, equivalently, absorbed solar radiation at the surface), but also of the surface temperature $T_{s}$. Equation (13) with $\beta_{s w}=\beta_{w}=0$ corresponds to the radiative equilibrium solution in a gray atmosphere. Note that (13) is not equal to (12) in the limit $k_{0} \rightarrow 0$.

\section{Radiation limits from stratospheric conditions}

We use water as the main condensable atmospheric gas with absorption in the infrared. Hence, the spectral limits of the atmospheric window are 8 and $12 \mu \mathrm{m}$ in wavelength (Kondratyev 1969). We adjust a cubic polynomial fit to $\beta_{w}(T)$ as a function of temperature and valid within the 200-600-K range (see Fig. 1). The coefficients of the cubic polynomial are -0.737774 , $0.00670592 \mathrm{~K}^{-1}, 1.39486 \times 10^{-5} \mathrm{~K}^{-2}$, and 9.02909 $\times 10^{-9} \mathrm{~K}^{-3}$, which produce an error smaller than $3 \%$. Calculations use $k_{1}=0.01 \mathrm{~m}^{2} \mathrm{~kg}^{-1}$ (Ingersoll 1969; Nakajima et al. 1992), and the vapor-liquid equilibrium curve for the condensable component follows that of water. Then, the saturation water vapor pressure $p_{c}^{*}$ is obtained from the Clausius-Clapeyron equation (see Nakajima et al. 1992)

$$
p_{c}^{*}(T)=p_{0}^{*} \exp \left(-\frac{l}{R T}\right),
$$

where $p_{0}^{*}=1.4 \times 10^{11} \mathrm{~Pa}, l$ is the latent heat of the condensable component $\left(=43655 \mathrm{~J} \mathrm{~mol}^{-1}\right)$, and $R$ is the universal gas constant $\left(=8.314 \mathrm{~J} \mathrm{~mol}^{-1} \mathrm{~K}^{-1}\right)$. The

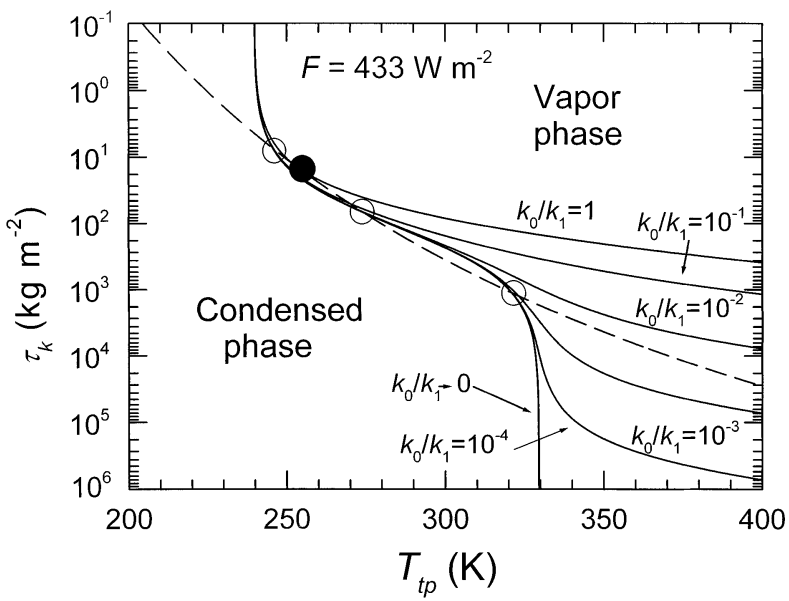

FIG. 2. Optical depth divided by the mass absorption coefficient $\tau_{k}$ as a function of the tropopause temperature $T_{\mathrm{tp}}$. The dashed line corresponds to the vapor-liquid equilibrium curve. Solid lines refer to radiative equilibrium curves with the same $\operatorname{OLR}\left(F=433 \mathrm{~W} \mathrm{~m}^{-2}\right)$ and for different levels of absorption in the atmospheric window (ratio $k_{0} / k_{1}$, where $k_{0}$ and $k_{1}$ are the mass absorption coefficients inside and outside the atmospheric window, respectively). The case with $k_{0} / k_{1}$ $=1$ is the gray assumption. The intersection points between both curves (circles) are states in both radiative and vapor-liquid equilibria.

mole fraction in a saturated troposphere is $x_{c}=p_{c}^{*} / p$, where $p$ is the total pressure. At the tropopause, $x_{c}=$ $p_{c}^{*}\left(T_{\mathrm{tp}}\right) / p_{\mathrm{tp}}$, where $T_{\mathrm{tp}}$ represents the tropopause temperature. From the above, we may express the coefficient $\tau_{k}$ [see the text below (12)] that appears in the equations for the tropopause temperature (12) and (13) as

$$
\tau_{k}\left(T_{t p}\right)=\frac{p_{c}^{*}\left(T_{t p}\right)}{g},
$$

since, for simplicity, we have assumed the same molecular weight for both condensable and noncondensable components (i.e., $m_{c}=m_{n}=\bar{m}$ ).

The coefficient $\tau_{k}$ is nothing but the optical depth divided by the mass absorption coefficient in each infrared band. Note that its value is independent of wavelength. The values of $T_{\mathrm{tp}}$ and $\tau_{k}$ that satisfy the vaporliquid equilibrium condition at the tropopause level [Eq. (15)] follow the dashed line shown in Fig. 2. Solid lines in Fig. 2 correspond to the values of $T_{\mathrm{tp}}$ and $\tau_{k}$ that satisfy the radiative equilibrium condition at the tropopause [Eq. (12)] for a fixed value of OLR $(\equiv F=$ $433 \mathrm{~W} \mathrm{~m}^{-2}$ ) and different values of the $k_{0} / k_{1}$ ratio. The solution for $k_{0} / k_{1}=1$ reverts to the classical gray case analyzed by several investigators (Ingersoll 1969; Goody and Yung 1989; Nakajima et al. 1992). Note that we do not include the values of $T_{\mathrm{tp}}$ and $\tau_{k}$ for $k_{0}=0$, since Eq. (13) also involves the surface temperature $T_{s}$ (i.e., we would need to specify the convective regime). This case (as well as those for $k_{0} \neq 0$ ) is analyzed in section 4 (radiation limits from tropospheric conditions). We also point out that the dashed line in Fig. 2 is not a function of the OLR $F$ (or, equivalently, of the ab- 


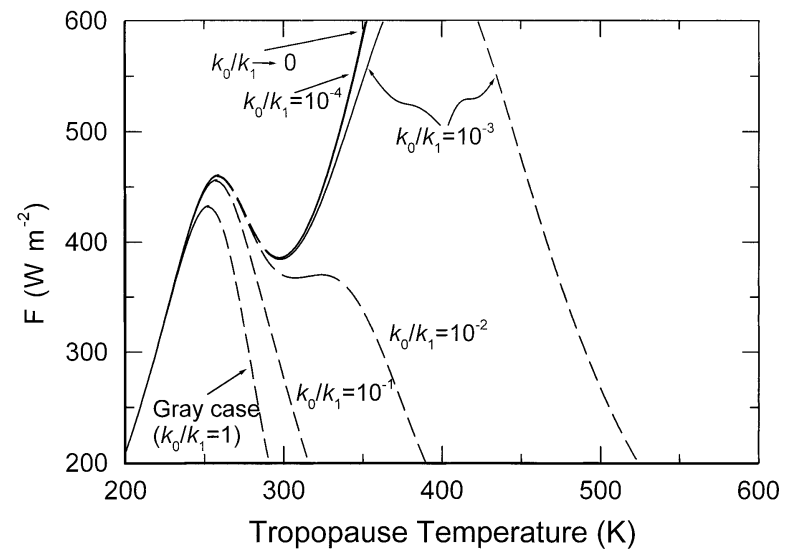

FIG. 3. Tropopause temperature for the states in both radiative and vapor-liquid equilibria as a function of the OLR $F$ for different levels of absorption in the atmospheric window. Solid (dashed) lines correspond to branches where an increase in the OLR leads to an increase (decrease) in the tropopause temperature.

sorbed solar radiation). In contrast, it is clearly seen from (12) that an increase of $F$ for a fixed value of $\tau_{k}$ leads to a higher value of $T_{\mathrm{tp}}$. This means that the solid curves in Fig. 2 move towards the right of the diagram as $F$ increases.

The intersection points between both dashed and solid curves correspond to values of $T_{\text {tp }}$ and $\tau_{k}$ at the tropopause in both radiative and vapor-liquid equilibria. The gray atmosphere shows only one solution (closed circle). In this case, the value of the OLR corresponds to the SKI limit since the solid curve for $F>F_{\text {SKI }}(=433$ $\mathrm{W} \mathrm{m}^{-2}$ ) would not intersect the dashed line at any point (the solid line would entirely lie in the vapor phase region of the diagram as discussed above). (The atmospheric radiation limits obtained from stratospheric conditions will now be referred to as SKI limits.) For values of OLR lower than $F_{\mathrm{SKI}}$, two possible solutions (i.e., two intersection points between the solid line and the dashed line) for the gray case would be obtained, though only one would be stable and attainable from an evolutionary viewpoint (Goody and Yung 1989). In contrast, three solutions in radiative as well as in vaporliquid equilibria may be obtained for a ratio $k_{0} / k_{1} \ll 1$. As an example, open circles show the three solutions found in Fig. 2 for the $k_{0} / k_{1}=10^{-3}$ case.

Figure 3 shows the $T_{\text {tp }}$ values as a function of the OLR for tropopause conditions that satisfy both radiative and vapor-liquid equilibria (e.g., as circles in Fig. 2 for $F=433 \mathrm{~W} \mathrm{~m}^{-2}$ ). Solid (dashed) lines in Fig. 3 indicate branches where the OLR increases when increasing (decreasing) $T_{\mathrm{tp}}$. Ingersoll (1969), Goody and Yung (1989), Nakajima et al. (1992), and Rennó (1997) argue that the dashed lines are unstable. Note the single SKI limit found for the gray case (also for the $k_{0} / k_{1}=$ $1 / 10$ case). In comparison, the case with $k_{0} / k_{1}=10^{-2}$ shows an absolute SKI limit at low $T_{\text {tp }}$ and a relative SKI limit at high ones. For a more transparent window (e.g., $k_{0} / k_{1}=10^{-3}$ ), the absolute SKI limit is at high $T_{\mathrm{tp}}$ and the relative one at low $T_{\mathrm{tp}}$.

From an evolutionary viewpoint, an increase in the incoming shortwave radiation over geologic time is expected from the fundamentals of star evolution (see, e.g., Crowley and North 1991). As a consequence, and since for simplicity we neglect changes in planetary albedo, the vertical axis in Fig. 3 may be understood as a nonlinear axis in time once the initial phase of planetary outgassing has finished, and the vapor-liquid equilibrium has been finally reached. Then, as time evolves, the tropopause temperature increases along the solid (stable) branch in the lower left corner of Fig. 3. The greenhouse runaway effect arises if the absorbed solar flux exceeds either the single SKI limit found in atmospheres with a very dirty window (e.g., $k_{0} / k_{1}=1$ ), or the absolute SKI limit found in atmospheres with a partially transparent window where the relative SKI limit appears at higher temperatures (e.g., $k_{0} / k_{1}=10^{-2}$ ). The climate state jumps to the second solid (stable) branch when the absorbed solar radiation exceeds the relative SKI limit and the absolute one is at higher temperatures (see the atmospheres with a very clean window, e.g., $k_{0} / k_{1}=10^{-3}$, in Fig. 3). Note that the vaporliquid equilibrium condition still holds in the new stable branch found at high values of tropopause temperatures.

\section{Radiation limits from tropospheric conditions}

The assumption that a convective regime sustains a pseudoadiabatic (or adiabatic) profile is only valid in an inviscid fluid (see Rennó and Ingersoll 1996). However, and for simplicity, we use a prescribed vertical temperature profile in a fully saturated troposphere. This empirical technique devised to characterize the atmospheric convective layer has been widely used in simple vertical models (e.g., Nakajima et al. 1992). The pseudoadiabatic vertical temperature gradient $\left(\gamma_{s}=d T / d p\right)$ follows (Iribarne and Godson 1981)

$$
\gamma_{s} \approx \frac{\frac{R T}{c_{p} p}+\frac{l}{c_{p} p} \frac{x_{c}}{x_{n}}}{1+\frac{c_{p v}}{c_{p}} \frac{x_{c}}{x_{n}}+\frac{l^{2}}{c_{p} R T^{2}} \frac{x_{c}}{x_{n}}},
$$

where $c_{p}(=3.5 R)$ and $c_{p v}(=4 R)$ are the mole specific heats at constant pressure of the noncondensable and condensable components, respectively; $x_{n}$ is the mole fraction of the noncondensable component $\left(=1-x_{c}\right)$. Implicitly in (16) is the assumption that condensed water precipitates immediately.

For a fixed value of the surface temperature $T_{s}$, we find the corresponding value of OLR $F$ (or absorbed solar radiation) using the following procedure. First, we choose an arbitrary value of the tropopause level $p_{\text {tp }}$, and the energy flux of radiation $F\left[=2 \pi \mu_{0}\left(I^{+}-I^{-}\right)\right]$ is computed from the integration over frequencies of 


$$
\begin{aligned}
I_{v}^{+}\left(p_{\mathrm{tp}}\right)= & B_{s v} \exp \left[\int_{p_{0}}^{p_{\mathrm{tp}}} k_{v} x_{c} m_{c} d p /\left(\bar{m} g \mu_{0}\right)\right] \\
& -\int_{p_{0}}^{p_{\mathrm{tp}}} \frac{k_{v} x_{c} m_{c} B_{v} d p}{\bar{m} g \mu_{0}} \\
& \times \exp \left[\int_{p}^{p_{\mathrm{tp}}} k_{v} x_{c} m_{c} d p^{\prime} /\left(\bar{m} g \mu_{0}\right)\right], \\
I_{v}^{-}\left(p_{\mathrm{tp}}\right)= & J_{v}\left(p_{\mathrm{tp}}\right)-\frac{F_{v}}{4 \pi \mu_{0}} .
\end{aligned}
$$

Equation (17a) follows from solving the radiative transfer equation (2) for the upward stream (Kourganoff 1952), where $p_{0}$ and $B_{s v}$ are the surface pressure and Planck function at the surface temperature, respectively. Equation (17b) follows directly from the definitions of $J_{v}$ and $F_{v}$ [see the text above (4)]. Note that the integration over pressure of the last term in the rhs of (17a) can be obtained once the tropopause level is fixed and the vertical distribution of temperatures is calculated from (16). The mole fraction of the condensable component $x_{c}$ in the troposphere is equal to $p_{c}^{*} / p$ where $p_{c}^{*}$ follows the saturation vapor pressure (14) (we assume a saturated troposphere). The integration of (17b) over frequencies is obtained from the value of $J$ deduced in (7) with (8) and (10). Then, we obtain a first value of $F$ at the tropopause deduced from $(17 \mathrm{a}-\mathrm{b})$.

On the other hand, the value of $T_{\mathrm{tp}}$ obtained by integrating (16) from $p_{0}$ to $p_{\text {tp }}$ is substituted in (12) [or (13)] to obtain a second value of $F$. The pressure level $p_{\text {tp }}$ is tuned until both values of $F$ coincide. For each value of $T_{s}$, a single value of $p_{\mathrm{tp}}$ is found.

\section{a. Atmosphere with water vapor only}

In this case, there is no noncondensable gas in the atmosphere (i.e., $x_{n}=0$, or equivalently, $p_{n 0}=0 \mathrm{~Pa}$, $p_{n 0}$ being the partial pressure of the noncondensable gas at the surface). Surface temperatures for the climate states in both radiative-convective and vapor-liquid equilibria are shown in Fig. 4 for different values of the $k_{0} / k_{1}$ ratio. The behavior for the gray case (i.e., $k_{0} / k_{1}=$ 1) agrees with that found by Nakajima et al. (1992), although the numerical values differ slightly, since we use the two-stream approximation instead of the Eddington's approach applied by Nakajima et al. (1992). The blackbody radiation emitted at the surface temperature $\left(=2 \mu_{0} \sigma T_{s}^{4}\right.$ within the two-stream approximation; see Liou 1992) is also plotted.

At low values of the surface temperature $T_{s}$, the atmosphere is so optically thin that the effect of the window is indistinguishable (all cases predict almost the same result in Fig. 4 at low $T_{s}$ ). In contrast, the dirtiness of the atmospheric window is a parameter of importance at high values of $T_{s}$. In this case, the atmosphere may be so optically thick that the OLR is insensitive to the

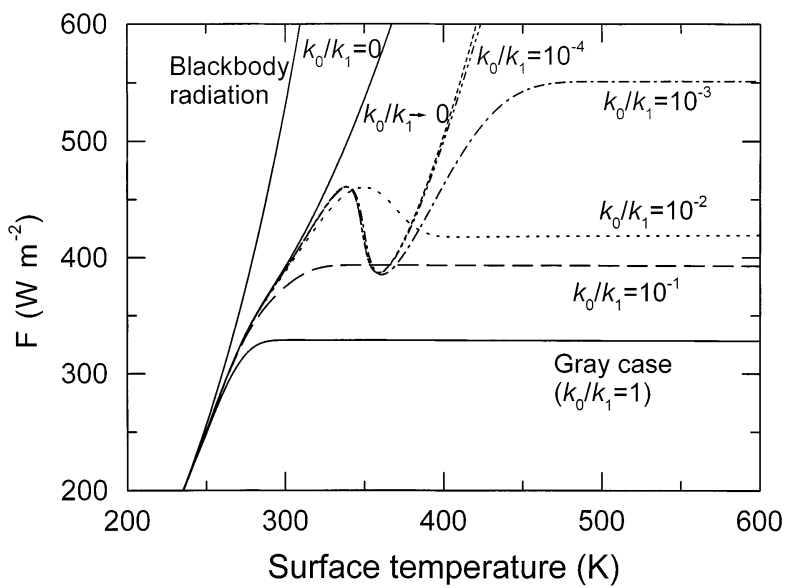

FIG. 4. OLR $F$ as a function of surface temperature in semigray atmospheres with fully saturated tropospheres. The degree of opacity of the atmospheric window is defined by the ratio $k_{0} / k_{1}$. A water vapor-only atmosphere is assumed. The blackbody radiation (i.e., emission in an atmosphere totally transparent to infrared radiation) is also shown.

structure of the lower atmospheric layers and, in particular, to $T_{s}$ (Kasting et al. 1984; Nakajima et al. 1992). This is the reason the solutions shown in Fig. 4 level out at high values of surface temperature. As Nakajima et al. (1992) have already pointed out, the radiation limit for a gray atmosphere is smaller than the absolute SKI limit found in Fig. 3. This is not the case for atmospheres with quasi-transparent windows at moderate values of surface temperature, since the value of the relative SKI limit found in Fig. 3 for ratios $k_{0} / k_{1} \leq 10^{-3}$ is the same as the relative limit obtained in Fig. 4. As a consequence, we find one of the main results of the present paper, which is the multiplicity of states for a fixed value of OLR $(=F)$ due to the nongray absorption.

In Fig. 4, the slope of the curves corresponds to the effective radiative damping coefficient $B_{\text {eff }}$ (i.e., $B_{\text {eff }}=$ $\partial F / \partial T_{s}$; see North et al. 1981), or to the inverse of the climatic sensitivity $\lambda$. From the stability theory applied to simple climate models, branches with a negative radiative damping coefficient $B_{\text {eff }}$ are classified as unstable (see, e.g., North et al. 1981, and the references therein). Note that on such branches, an increase of the absorbed solar radiation at the surface (or, equivalently, OLR) would imply an unexpected decrease of the surface temperature. Branches with unstable solutions are, therefore, unattainable for the real climate system. For a given value of OLR, two stable and one unstable solutions may be found depending on the value of the $k_{0} / k_{1}$ ratio. A single (stable) solution not bounded is found for an atmosphere with null absorption within the atmospheric window.

\section{b. Atmosphere with two components}

In this case, the partial pressure of the noncondensable (and transparent) ideal gas at the surface $p_{n 0}$ equals 


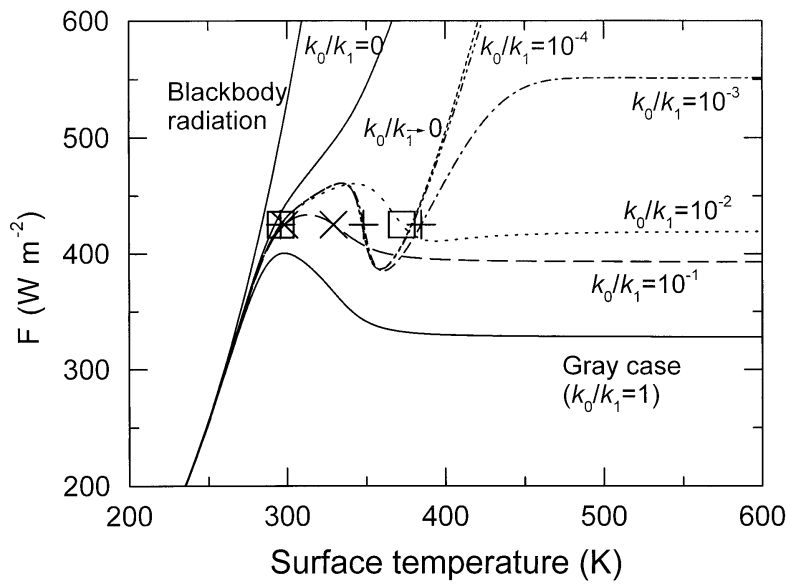

FIG. 5. As in Fig. 4, except for an atmosphere with a partial pressure of the noncondensable and transparent component at the surface equal to $10^{5} \mathrm{~Pa}$. Symbols denote the states whose vertical structure is detailed in Fig. 6.

$10^{5} \mathrm{~Pa}$, roughly that of the earth's current state. Results of the OLR as a function of the surface temperature are shown in Fig. 5. (Symbols at the $425 \mathrm{~W} \mathrm{~m}^{-2}$ level show the states whose vertical structures are depicted in Fig. 6.)

The introduction of a noncondensable gas reduces the atmospheric opacity at low temperatures since it increases the lapse rate (16). Then, the OLR for a fixed value of $T_{s}$ is higher than in an atmosphere with water vapor only (compare Figs. 4 and 5 at low values of $T_{s}$ ). For very high values of surface temperature $T_{s}$, the partial pressure of water vapor will exceed that of the noncondensable component. Thus, the two component atmosphere would invariably behave like a water vaporonly atmosphere (compare Figs. 4 and 5 at high values of $T_{s}$ ). Therefore, the intermediate radiation limit found in Fig. 5 (and not in Fig. 4) for atmospheres with opaque windows is due to the influence of the noncondensable component on the tropospheric lapse rate. Then, the maximum value for this radiation limit would be obtained by applying the dry adiabatic lapse rate (see, Nakajima et al. 1992). It turns out that this radiation limit is greater than the SKI one from the values of the parameters used in the present model. Therefore, an atmosphere with a very high partial pressure for the noncondensable gas (greater than $10^{5} \mathrm{~Pa}$ ) would reach the SKI limit shown in Fig. 3 at intermediate values of surface temperatures, but would tend to the asymptotic limit shown in Fig. 4 at high values of $T_{s}$.

Figure 6 depicts the vertical structure of the atmosphere for those states denoted by symbols in Fig. 5. Closed circles indicate the tropopause level, shown in $\mathrm{km}$. All the profiles tend to the same TOA temperature $(\approx 238.7 \mathrm{~K})$, since the OLR is equal to $4 \mu_{0} \sigma T^{4}$ within the two-stream approximation (Liou 1992). For the $k_{0} /$ $k_{1}=10^{-1}$ case, the atmosphere is so optically thick that tropopause temperatures lie in the solid line branch in

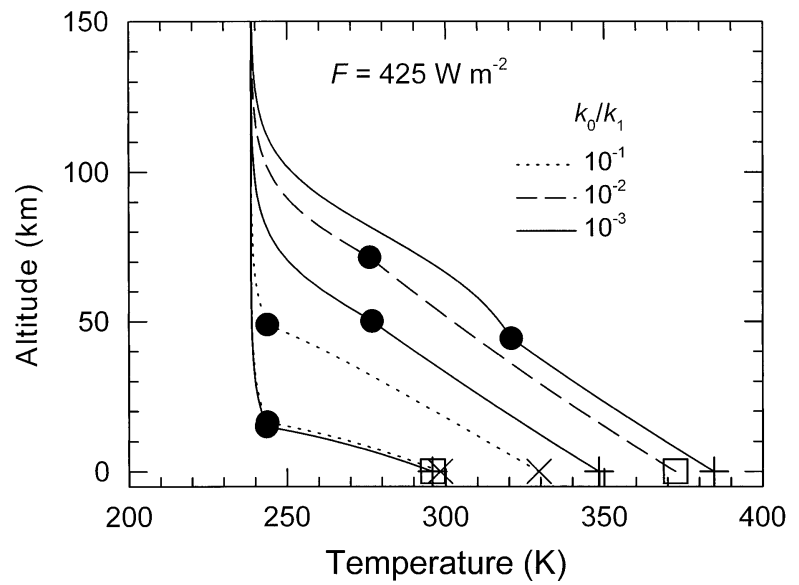

FIG. 6. Vertical temperature profiles of semigray atmospheres with different degree of opacity within the atmospheric window $\left(k_{0} / k_{1}\right.$ ratio). Symbols at the surface correspond with those shown in Fig. $5\left(F=425 \mathrm{~W} \mathrm{~m}^{-2}\right)$. Closed circles indicate the tropopause level.

Fig. 3 only. The low amount of absorber within the atmospheric window in the $k_{0} / k_{1}=10^{-2}$ case thins the atmosphere and permits two different tropopause temperatures for the same amount of OLR, the highest being in the unstable branch of Fig. 3. Three different profiles with three different tropopause values are found for the $k_{0} / k_{1}=10^{-3}$ case. Note that profiles with an unstable tropopause temperature ( $T_{\mathrm{tp}}$ in dashed branches in Fig. 3) also lead to a surface temperature with a negative $B_{\text {eff }}$ in Fig. 5. In these cases, the lapse rate just below the tropopause is smaller than that just above.

We stress the high variation of stratospheric temperatures (up to $82 \mathrm{~K}$ in the highest $T_{s}$ profile for the $k_{0} /$ $k_{1}=10^{-3}$ case shown in Fig. 6) occurred when the tropopause level reached the second stable branch in Fig. 3. In the gray case, the variation in the unique branch is so small that the approximation of an isothermal stratosphere is valid (Kasting 1988). However, this assumption cannot hold in some semigray atmospheres.

\section{Discussion}

The simplifications assumed in the present model exclude a quantitative analysis of the results. A more physically based model should include a treatment of the water vapor as a nonideal gas, use a nonsimplified tropospheric lapse rate, and, for example, include the critical point of the water vapor (e.g., Abe and Matsui 1988; Kasting 1988). Note, also, that the continuum absorption of water vapor within the atmospheric window is proportional to its partial pressure (Kasting et al. 1984), so the ratio $k_{0} / k_{1}$ is not constant but varies with temperature. At high values of surface temperature the atmospheric window would close so the solution would tend to the gray case.

A planetary atmosphere with a transparent window 


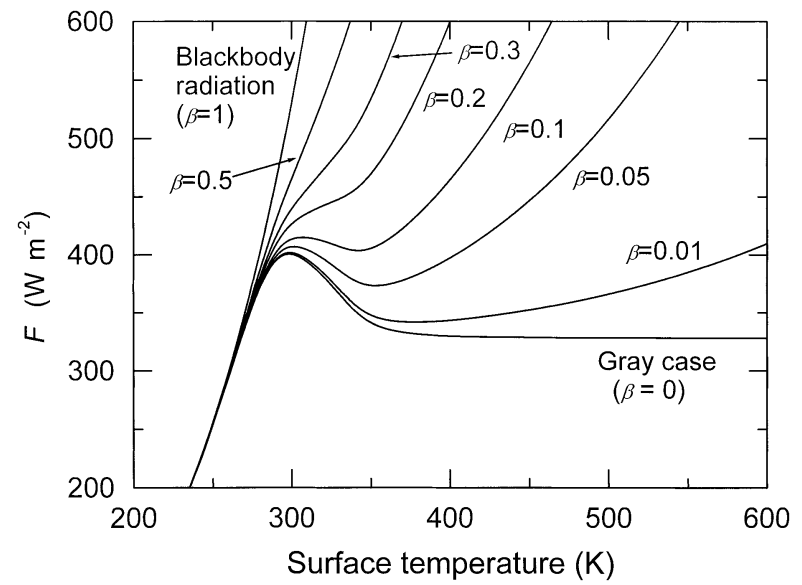

FIG. 7. OLR $F$ as a function of surface temperature in semigray atmospheres with fully saturated tropospheres. The atmospheric window, with an effective width $\beta_{w}$, is totally transparent to infrared radiation $\left(k_{0}=0\right)$. The partial pressure of the noncondensable transparent component at the surface is equal to $10^{5} \mathrm{~Pa}$.

(i.e., $\left.k_{0}=0\right)$ may also exhibit a finite runaway effect, though not an infinite one. Figure 7 shows the OLR as a function of the surface temperature for atmospheres with $k_{0}=0, p_{n 0}=10^{5} \mathrm{~Pa}$, and different values of $\beta_{w}$ (constant). A multiplicity of stable states (i.e., of branches with positive $B_{\text {eff }}$ ) may be found for a number of "infrared holes" in the atmosphere smaller than a threshold value. In this case, the finite runaway effect depends on the tropospheric structure, so a water vaporonly atmosphere does not show such a multiplicity of states. For $\beta_{w} \approx 0.3$, a single not-bounded solution is found (see Fig. 7), which agrees with Simpson (1928) and Weaver and Ramanathan (1995), since these authors use a transparent window.

The multiplicity of states shown in Figs. 4, 5, and 7, resembles the classical multiple equilibria obtained from simple energy balance models (EBMs) when including the ice albedo feedback (see, e.g., North et al. 1981). However, both types of solutions are essentially different. In EBMs, the multiplicity of steady states refers to global conditions (i.e., the entire planet is either in one stable branch or in another), whereas in the present onedimensional radiative-convective model, the equator may be in one stable branch and the poles in another. Note that different branches not only differ in the values of their surface temperature (see Fig. 5), but also in the depth of their convective layers. Figure 8 shows the height of the tropopause $(\mathrm{km})$ as a function of the OLR emitted at TOA for the cases shown in Fig. 5. For the sake of argument, let us assume an atmosphere with low absorption within the atmospheric window (for example, $k_{0} / k_{1}=10^{-3}$ ). Then, the equatorial atmosphere would jump to the second stable branch shown in Fig. 5 , if the absorbed solar radiation exceeded the relative SKI limit found in Fig. 3. However, no change would be found if the absorption in polar regions was below the SKI limit. In this case, tropical and polar tropopause

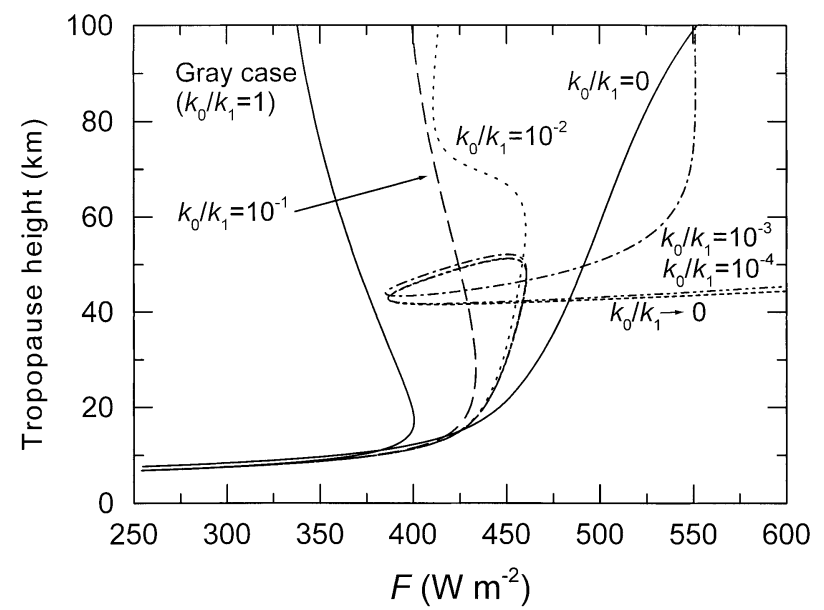

FIG. 8. Tropopause heights as a function of the OLR $F$ for the results shown in Fig. 5. Closed circles shown in Fig. 6 correspond to heights at $F=425 \mathrm{~W} \mathrm{~m}^{-2}$.

heights would be substantially different (see Fig. 8). In the previous scenario we have neglected dynamical effects, which would be of particular importance by exporting heat from the Tropics to the extratropics.

For the earth's current state, the ratio $k_{0} / k_{1}$ is about $10^{-2}$ (see Kasting et al. 1984), and the equator would be in a "local" runaway state (see Pierrehumbert 1995). (The condition of local runaway state cannot be directly deduced from Fig. 4 and the annual mean equatorial absorption, since our model was derived for qualitative purposes only.) Pierrehumbert (1995) suggests that dry tropical regions maintained by subsidence reduce the tropical sensitivity and keep the tropical atmosphere from falling into a runaway state. Indeed, the stabilizing effect of these dry pools can be seen from Figs. 5 and 7 , here used as stability charts. Thus, the effective radiative damping coefficient $B_{\text {eff }}$ (or the slope of the curves in Figs. 5 and 7) is higher in dry (transparent) conditions (analogous to $k_{0} / k_{1}=0$ in Fig. 5, or to high $\beta_{w}$ values in Fig. 7) than in wet (opaque) ones (analogous to $k_{0} / k_{1}=1$ in Fig. 5 , or to low $\beta_{w}$ values in Fig. 7).

\section{Conclusions}

We have developed a simplified radiative-convective model to investigate the effects of the nongray absorption in the radiation limits that may exhibit a planetary atmosphere. The model follows that of Nakajima et al. (1992), but including an atmospheric window in the infrared spectrum. This simple modification leads to completely new results, summarized as follows.

1) The single (absolute) SKI limit found in gray atmospheres is not obtained in nongray atmospheres with fully transparent infrared regions. A relative radiation limit may be found for atmospheres with very narrow windows (see Fig. 7). However, the 
longwave radiation emitted by any nongray atmosphere with fully transparent infrared regions is not bounded (i.e., the infinite runaway greenhouse point does not exist; see also Fig. 7).

2) Relative and absolute SKI limits are found in atmospheres with low (but finite) absorption within the atmospheric window (see Fig. 3). The atmosphere experiences a finite runaway effect when the absorbed solar radiation exceeds the relative SKI limit. Exceeding the absolute SKI limit would lead to an infinite runaway effect.

3) SKI limits arise, by definition, from stratospheric conditions (i.e., from the consistency between the vapor-liquid equilibrium condition and the pure radiative equilibrium one; see Figs. 2-3). However, radiation limits obtained in a radiative-convective atmosphere may be smaller than the SKI limits (see Nakajima et al. 1992). The asymptotic limit of the OLR, reached at very high values of surface temperature in a radiative-convective atmosphere with a fully saturated troposphere and a noncondensable and transparent gas that exerts a partial pressure of $10^{5} \mathrm{~Pa}$ at the surface, is smaller than the absolute SKI limit (compare Fig. 5 with the radiation limits in Fig. 3).

4) One of the main results of the present paper is the finding of a multiple solution in semigray radiativeconvective atmospheres (see Figs. 4-5). Stable branches differ in their tropopause temperatures and heights. The multiplicity of stable states allows for the existence of a warm ocean in a very opaque atmosphere and a small value of absorbed solar radiation.

Under conditions of multiple stable equilibria, the surface temperature follows a hysteresis-like cycle as a function of the OLR. Since we assume a steady increase of OLR as a function of time (neglect cloud effects and changes in albedo), we implicitly reject the existence of an oscillatory behavior of the system with an alternation of states from different stable branches.

Multiple equilibria in a simple vertical model has been recently obtained by assuming a surface albedo feedback (e.g., Li et al. 1997). These results, however, are essentially different to those found here. We have shown that the nongray absorption by itself may generate multiple stable states, and therefore, identical surfaces (emitting the same OLR) may lie on different stable branches. Our results also differ from the intriguing multiple equilibria found in radiative-convective models with hydrological cycle (Rennó 1994, 1997), as well as from the bimodal solutions recently obtained in a pure radiative equilibrium model by Ide et al. (2001). We suspect that the multiplicity of stable states here found has not been previously revealed since the semigray atmosphere for the earth's current state (i.e., $\beta$ $\approx 0.3, k_{0} / k_{1} \approx 1 / 100$ ) predicts a single stable state with surface temperatures smaller than $380 \mathrm{~K}$ (see Fig. 5).
Then, the nongray absorption by itself may not be enough to produce a multiple set of stable solutions for the earth's current state. Nevertheless, and besides revealing a fundamental behavior of nongray atmospheres, the multiple equilibria here obtained may still be of particular importance for other planetary atmospheres.

Acknowledgments. T. Pujol gratefully acknowledges the support of a Fulbright-Generalitat de Catalunya postdoctoral grant, as well as the partial support of the Ministerio de Ciencia y Tecnologia of the Spanish government under Contract REN 2000-1621 CLI.

\section{REFERENCES}

Abe, Y., and T. Matsui, 1988: Evolution of an impact-generated $\mathrm{H}_{2} \mathrm{O}-$ $\mathrm{CO}_{2}$ atmosphere and formation of a hot proto-ocean on earth. J. Atmos. Sci., 45, 3081-3101.

Arking, A., and K. Grossman, 1972: The influence of line shape and band structure on temperatures in planetary atmospheres. $J$. Atmos. Sci., 29, 937-949.

Chandrasekhar, S., 1960: Radiative Transfer. Dover, 393 pp.

Crowley, T. J., and G. R. North, 1991: Paleoclimatology. Oxford University Press, 349 pp.

Emanuel, K. A., 1994: Atmospheric Convection. Oxford University Press, $580 \mathrm{pp}$.

Goody, R. M., and Y. L. Yung, 1989: Atmospheric Radiation. Theoretical Basis. 2d ed. Oxford University Press, 519 pp.

Houghton, J. T., 1986: The Physics of Atmospheres. 2d ed. Cambridge University Press, $271 \mathrm{pp}$.

Hu, Q., and D. A. Randall, 1995: Low-frequency oscillations in radiative-convective systems. Part II: An idealized model. J. Atmos. Sci., 52, 478-490.

Ide, K., H. Le Treut, Z.-X. Li, and M. Ghil, 2001: Atmospheric radiative equilibria. Part II: Bimodal solutions for atmospheric optical properties. Climate Dyn., 18, 29-49.

Ingersoll, A. P., 1969: The runaway greenhouse: A history of water on Venus. J. Atmos. Sci., 26, 1191-1198.

Iribarne, J. V., and W. L. Godson, 1981: Atmospheric Thermodynamics. 2d ed. Kluwer Academic, 259 pp.

Kasting, J. F., 1988: Runaway and moist greenhouse atmospheres and the evolution of Earth and Venus. Icarus, 74, 472-494.

—_ J. B. Pollack, and T. P. Ackerman, 1984: Response of Earth's atmosphere to increases in solar flux and implications for loss of water from Venus. Icarus, 57, 335-355.

Kelly, M. A., D. A. Randall, and G. L. Stephens, 1999: A simple radiative-convective model with hydrological cycle and interactive clouds. Quart. J. Roy. Meteor. Soc., 125, 837-869.

Komabayasi, M., 1967: Discrete equilibrium temperatures of a hypothetical planet with the atmosphere and the hydrosphere of one component-two phase system under constant solar radiation. J. Meteor. Soc. Japan, 45, 137-138.

Kondratyev, K. YA., 1969: Radiation in the Atmosphere. Academic Press, 912 pp.

Kourganoff, V., 1952: Basic Methods in Transfer Problems. Oxford University Press, $281 \mathrm{pp}$.

Li, Z.-X., K. Ide, H. Le Treut, and M. Ghil, 1997: Atmospheric radiative equilibria in a simple column model. Climate Dyn., 13, $429-440$.

Lindzen, R. S., A. Y. Hou, and B. F. Farrell, 1982: The role of convective model choice in calculating the climate impact of doubling $\mathrm{CO}_{2}$. J. Atmos. Sci., 39, 1189-1205.

—-, M.-D. Chou, and A. Y. Hou, 2001: Does the earth have an adaptive infrared iris? Bull. Amer. Meteor. Soc., 82, 417-432.

Liou, K. N., 1992: Radiation and Cloud Processes in the Atmosphere. Oxford University Press, 487 pp. 
Manabe, S., and F. Möller, 1961: On the radiative equilibrium and heat balance of the atmosphere. Mon. Wea. Rev., 89, 503-532.

- and R. F. Strickler, 1964: Thermal equilibrium of the atmosphere with a convective adjustment. J. Atmos. Sci., 21, 361385 .

, and R. T. Wetherald, 1967: Thermal equilibrium of the atmosphere with a given distribution of relative humidity. J. Atmos. Sci., 24, 241-259.

Nakajima, S., Y.-Y. Hayashi, and Y. Abe, 1992: A study on the "runaway greenhouse effect" with a one-dimensional radiative-convective equilibrium model. J. Atmos. Sci., 49, 2256-2266.

North, G. R., R. F. Cahalan, and J. A. Coakley Jr., 1981: Energy balance climate models. Rev. Geophys. Space Phys., 19, 91-121.

Pierrehumbert, R. T., 1995: Thermostats, radiator fins, and the local runaway greenhouse. J. Atmos. Sci., 52, 1784-1806.

Pollack, J. B., 1971: A nongrey calculation of the runaway greenhouse: Implications for Venus' past and present. Icarus, 14, 295 306.

Ramanathan, V., and J. A. Coakley Jr., 1978: Climate modeling through radiative-convective models. Rev. Geophys. Space Phys., 16, 465-489.

Rennó, N. O., 1994: Multiple equilibria in radiative-convective atmospheres. Proc. AGU Fall Meeting, Washington, DC, Amer. Geophys. Union, 129-130.

_ 1997: Multiple equilibria in radiative-convective atmospheres. Tellus, 49A, 423-438.

- , and A. P. Ingersoll, 1996: Natural convection as a heat engine: A theory for CAPE. J. Atmos. Sci., 53, 572-585.

Simpson, G. C., 1927: Some studies in terrestrial radiation. Mem. Roy. Meteor. Soc., 16, 69-95.

_ 1928: Further studies in terrestrial radiation. Mem. Roy. Meteor Soc., 21, 1-27.

Thomas, G. E., and K. Stamnes, 1999: Radiative Transfer in the Atmosphere and Ocean. Cambridge University Press, 517 pp.

Weaver, C. P., and V. Ramanathan, 1995: Deductions from a simple climate model: Factors governing surface temperature and atmospheric thermal structure. J. Geophys. Res., 100, 11 585-11 591. 